\title{
REDUZIERUNG VON MR-BILDARTEFAKTEN DURCH GEZIELTE KOM- BINATION PARA- UND DIAMAGNETISCHER MATERIALIEN
}

\author{
E. Anders \\ Konstruktion, Mikro- und Medizintechnik, Technische Universität Berlin, Deutschland \\ e.anders@berlin.de
}

\begin{abstract}
A theoretical mathematical model of dualcomponent paramagnetic and diamagnetic material to cancel metal-induced magnetic resonance imaging (MRI) artifacts was investigated and evaluated. The magnetization produced by material causes gradient linearity distortion. By using materials with opposing paramagnetic and diamagnetic properties it is supposed to compensate these effects.

Therefore the interferences by paramagnetic and diamagnetic materials on images in a closed 1.5 Tesla high field magnetic resonance imaging system was experimentally examined for different types of sequences and correlated to their magnetic susceptibility. Basing on this results the concept of dual-component materials to solve the problems of MRI-artifacts was theoretically checked by using the finite element method and experimentally in the MRI.
\end{abstract}

Keywords-MRI, artifacts, compensation, susceptibility, dual-component materials

\section{Einleitung}

Die Magnetresonanztomographie (MRT) hat sich in den letzten Jahren zu einem immer beliebteren Mittel der bildgebenden Diagnostik entwickelt. Die Voraussetzung für ein präzises Abbild des Meßobjektes ist ein starkes, homogenes Magnetfeld. Werden die Feldlinien aber verzerrt, hat das in diesen Bereichen eine Veränderung des Signals und somit eine Verfälschung der Aufnahme zur Folge. Bildunregelmäßigkeiten, die nicht der Realität entsprechen, werden Artefakte genannt.

Es ist fast unumgänglich Hilfsmaterialien bei Messungen im $M R T$ zu verwenden, aber gerade diese Materialien üben Einfluß auf das Magnetfeld aus und können daher die Ursache für Artefakte und Fehldiagnosen sein. Es ist also notwendig $M R$-kompatible Materialien zu finden.

Unterschiedliche Substanzen haben in Abhängigkeit von ihrer Suszeptibilität verschiedenartige Effekte auf Magnetfelder. Paramagnetische und diamagnetische Materialien zeigen eine entgegengesetzte Wirkung auf den Feldlinienverlauf. Dieser Umstand bildet die Grundlage für einen neuen theoretischen Ansatz zur Kompensation der Feldverzemungen.

Ziel der Arbeit war, diesen Ansatz zu überprüfen und eine gecignete Kombination von para- und diamagnetischen Werkstoffen ausfindig zu machen, die zu einem Ausgleich der von ihnen verursachten Feldinhomogenitäten führt.

\section{Materialien}

Alle Messungen sind in cinem geschlossenen 1,5-TeslaHochfeld-Tomographen der Firma Philipps (Typ: Gyro- scan ACS-NT) vorgenommen worden. Die Untersuchungen erfolgten mit drei verschiedenen, in klinischen Untersuchungen häufig verwendeten Sequenzen [Spin-Echo(T1); Gradienten-Echo-(FFE); Echo-Planar-Imaging (EPI)] deren wichtigsten Sequenzparameter in Tabelle 1 aufgeführt sind.

Tabelle 1: Sequenztypen und -parameter

\begin{tabular}{lccccc}
\hline Sequenz & $\begin{array}{c}\text { TE } \\
{[\mathrm{ms}]}\end{array}$ & $\begin{array}{c}\text { TR } \\
{[\mathrm{ms}]}\end{array}$ & $\begin{array}{c}\text { Flip-Angle } \\
{\left[{ }^{\circ}\right]}\end{array}$ & $\begin{array}{c}\text { Schichtdicke } \\
{[\mathrm{mm}]}\end{array}$ & $\begin{array}{c}\text { Scanzeit } \\
{[\mathrm{min}]}\end{array}$ \\
\hline T1 & 15 & 216 & 90 & 5 & $2: 59$ \\
FFE & 4,6 & 7,8 & 25 & 5 & $0: 27$ \\
EPI & 9,3 & 9,3 & 31 & 5 & $0: 26$ \\
\hline
\end{tabular}

Die Bilddarstellungen im MRT erfolgte durch Zuhilfenahme einer mit physiologischer Flüssigkeit $(0,011 \mathrm{mmol}$ $\mathrm{Gd} / \mathrm{i} ; 0,157 \mathrm{mmol} \mathrm{Mn}^{2+} /$ ) gefüllter Kunststoffbox (Phantom). Das Phantom wurde fest auf einer Grundplatte aus PMMA montiert. Diese wurde mit einer, vom Phantom ausgehenden Wegmeßskalienung versehen. Zylindrische Probenstücke verschiedenster Materialien, deren Einfluß auf die Bildqualität unterschiedlicher Meßsequenzen bestimmt werden sollte, wurden auf einer Schaumstoffauflage befestigt und konnten so, gegen unerwünschte Bewegungen gesichert, in variierenden Entfernungen zum Phantom, auf die Grundplatte gebracht werden.

Die Suszeptibilitätsbestimmung der Materialien erfolgte mit einer Suszeptibilitätswaage von JME, die in Anlehnung an die Gouy-Waage entwickelt wurde.

Berechnungen und Auswertungen wurden mit einer Shareware Version des finite Elemente Programms FEMM (Finite Element Method Magnetics) Version 3.0 durchgeführt.

\section{Methoden}

Der Meßaufbau wurde in zwei Orientierungen zum statischen Magnetfeld in den MRT gelegt War die Probe parallel zur Feldrichtung positioniert, wurde ein sagittaler Scan durchgefuihrt, bei senkrechter Ausrichtung ein transversaler.

Zunächst wurden die Auswirkungen gängiger para- und diamagnetischer Materialien mit unterschiedlichen Suszeptibilitäten auf dic Bildgcbung im MRT crforscht. Bei den Messungen mit diesen Proben wurde das auf dem Kissen befestigte Werkstïck runächst dirckt an das Phantom gelegt und dann durch Zuhilfenahme der aufgeklebten Skala in je nach Artfaktintensität varicrenden Abständen parallel so weit von ihm entfernt, his die $A_{r}$ tefakte verschwanden. Dic so erhaltenen Bilder wurden 
dann verschiedenartig ausgewertet. Um objektive und nachvollzichbare Ergebnisse zu crhalten, mußten dic Suszeptibilitäten der Werkstoffe bestimmt werden, so da $B$ dic verursachten $A$ rtefakte im Zusammenhang mit dieser spezifischen Eigenschaf evaluiert werden konnten. Diese Betrachtungen licferten Grundlage und Eckdaten für dic weiterfülırenden Analysen. Es konnte beurtcilt werden welche der Materialien die besseren Voraussetzungen für den eigentlichen Kern der Arbeit erfüllen und eine erste Abschätzung der Erfolgschancen getroffen werden. Der Forderung, Werkstoffe so zu kombinicren, daß sic aufgrund entgegen wirkender Kräfte ihre Gcsamtsuszeptibilität so kompensieren, daß diese keinen Einfluß mehr auf die Umgebung nimmt, kann am leichtesten genüge getan werden, wenn man sich an einfache gcometrische Formen hält.

Paramagnetische und diamagnetische Materialien haben die Eigenschaft die Feldlinien in entgegengesetzter Richtung zu verzerren. Es bietet sich also an, diesen Effekt zu nutzen und Werkstoffe mit solchen Eigenschaften sinnvoll zu kombinieren. Die einfachste Art ist, einen Hohlzylinder des einen Werkstoffes mit einem $\mathrm{Zy}$ linder des anderen zu füllen.

„Die für ein NMR-Experiment relevanten Magnetfeldinhomogenitäten $\Delta \mathrm{B}_{\mathbf{z}}$, die durch ein unendlich langen Zylinder hervorgerufen werden, dessen Achse quer zu den Feldlinien eines zuvor homogenen Magnetfeldes der Flußdichte $\mathrm{B}_{0} \mathrm{e}_{\mathrm{z}}$ liegt (Abb.1) und dessen magnetische Suszeptibilität $\chi_{2}$ sich von der Suszeptibilität $\chi_{1}$, des ihn umgebenden Stoffes unterscheidet berechnen sich zu“ [1]:

$$
\frac{\Delta B_{z}}{B_{0}}=\delta_{l, z} \cdot R_{i}^{2} \frac{y^{2}-z^{2}}{\left(y^{2}+z^{2}\right)^{2}}
$$

mit:

$$
\delta_{l, z}:=\frac{\chi_{1}-\chi_{z}}{2+\chi_{1}+\chi_{z}}
$$

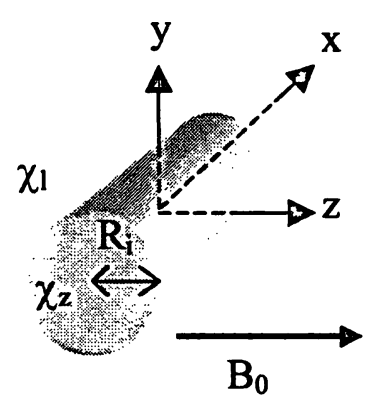

Abbildung 1: Zylinder im Magnetfeld

Wird dieser Zylinder in ein zweites Material mit der Suszeptibilität $\chi_{\mathrm{a}}$ eingehüllt (Abb. 2), berechnen sich die nun auftretenden Magnetfeldinhomogenitäten mit:

$$
\begin{aligned}
& \frac{\Delta B_{z}}{B_{0}}=\delta_{l, z} \cdot R_{t}^{2} \frac{y^{2}-z^{2}}{\left(y^{2}+z^{2}\right)^{2}} \\
& \text { mit: } \\
& y^{2}+z^{2}>R_{l}^{2} \\
& \delta_{l, z}:=\frac{R_{a}^{2} \cdot \delta_{l, a}+R_{l}^{2} \cdot \delta_{a, i}}{R_{a}^{2}+R_{l}^{2} \cdot \delta_{a, i} \cdot \delta_{l, a}} \\
& \chi_{1}
\end{aligned}
$$

Abbildung 2: Querschnitt Doppelzylinder

Hätte der in Abbildung 1 gezeigte Zylinder dieselbe Suszeptibilität $\left(\chi_{2}\right)$ wie seine Umgebung $\left(\chi_{1}\right)$, hätte dies zur Folge, daß die Inhomogenitäten $\Delta \mathrm{B}_{\mathbf{z}}$ (Gl.1 und 2) verschwinden. Nimmt man an, daß dieser $Z y-$ linder aus einem Doppelzylinder besteht (Abb. 2), würden sich die auftretenden Magnetfeldinhomogenitäten mit den Gleichungen 3 und 4 berechnen lassen. Aus der Forderung nach einem Verschwinden der Inhomogenitäten folgt für $\delta_{l, z}, \mathrm{da}$ es einen Wert von Null annimmt.

Diese Überlegungen führen zu der Bedingungsgleichung:

$$
\frac{R_{a}^{2}}{R_{i}^{2}}=\frac{\left(\mu_{i}-\mu_{a}\right) \cdot\left(\mu_{l}+\mu_{a}\right)}{\left(\mu_{l}-\mu_{a}\right) \cdot\left(\mu_{i}+\mu_{a}\right)}
$$

\section{(R: Radius; $\mu$ : magnetische Permeabilität;}

Index: i: Kernmaterial; a: Mantelmaterial; l: Luft)

Der nächste wichtige Schritt war die Kontrolle des theoretischen Ansatzes mit einer neuen, möglichst genauen Methode. $\mathrm{Zu}$ diesem $\mathrm{Zweck}$ wurde die Finite Elemente Methode herangezogen, mit der dieser Ansatz bestätigt, aber auch die Schwachstellen und Probleme für dessen Realisierung aufgezeigt werden konnten. Mit diesem Wissen und den Ergebnissen aus den Voruntersuchungen sind eigene Ansätze gemacht und mit der Finiten Elemente Methode kalkuliert worden. Diese neu berechneten Materialkombinationen wurden daraufhin im $M R T$ untersucht und ausgewertet.

\section{Ergebnisse}

Die Untersuchung verschiedener Materialien im MRT hat ergeben, daß schon kleine Permeabilitätsunterschiede zwischen Werkstoff und Umgebung zu großen 
Bildartefakten in Form von Aufhellungen, Verdunkelungen und sogar Konturverzerrungen als auch Signalauslöschungen führen können. In der Abbildung 3 sind die Aufnahmen des Phantoms ohne Zugabe eines Werkstoffes bei den verwendeten Sequenzen gezeigt (Referenzmessungen).

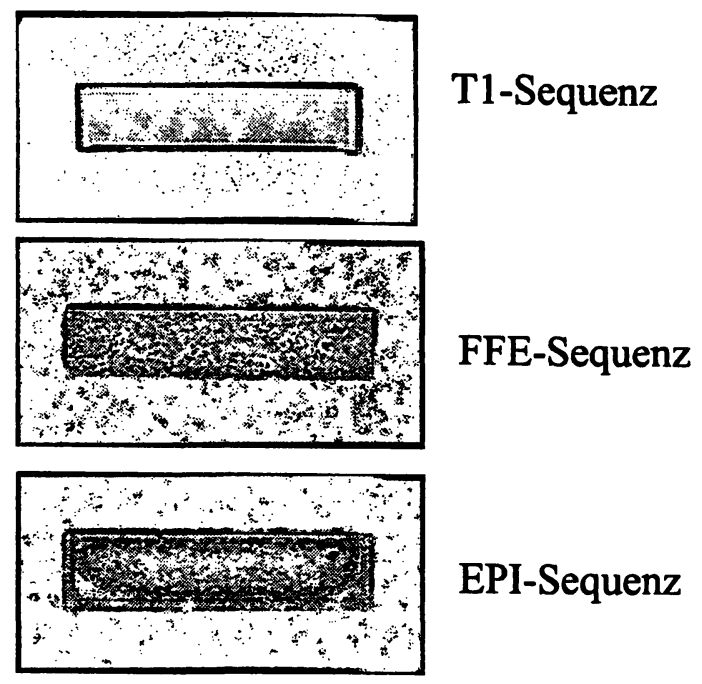

Abbildung 3: Referenzmessungen

Die Abbildung 4 zeigt Beispiele von verursachten Artefakten.
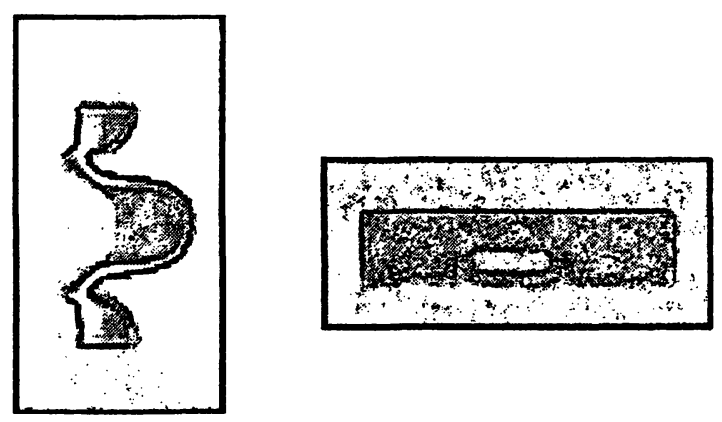

Abbildung 4: Artefakte

Es konnte gezeigt werden, daß die von den Materialien verursachten Feldinhomogenitäten, die ausschlaggebend für die Artefakte sind, unterschiedlichen Faktoren obliegen. Zum einen sind die allgemeinen Materialspezifikationen, wie Suszeptibilität, Leitfähigkeit und Geometrie, zum anderen ist aber auch die Orientierung im Magnetfeld von entscheidender Bedeutung.

Weiterhin waren einige Kompensationserfolge zu verzeichnen. Leider stellte sich heraus, daß das verfolgte Ziel einer vollständigen Kompensation der Feldinhomogenitäten mit diesem Ansatz nur schwer wenn überhaupt zu erreichen ist.

Für sichtbare Artefakte konnte nachgewiesen werden, daß aufgehellte Bereiche schlechter als verdunkelte und Feldinhomogenitäten, die breit gestreut sind und somit recht homogene und großflächigen Artefakte erzeugen, ebenfalls nicht so leicht, wie von kleinen Feldspitzen hervorgerufene Bildveränderungen zu erkennen sind.
Die Untersuchung der verwendeten Sequenzen ergab, daß die T1-Sequenz die beste Abbildungsqualität und die geringste Empfindlichkeit auf Feldinhomogenitäten aufwies, während die EPI-Sequenz die schlechtesten Ergebnisse erzielte. Aufgrund der hohen Bildgüte der T1-Sequenz waren bei ihr die erzeugten Artefakte allerdings auch am besten zu erkennen. Sie antwortet auf starke Feldverzerrungen mit den intensivsten Konturverzerrungen. Die FFE-Sequenz reagiert ein wenig sensibler auf Feldinhomogenitäten und hat eine etwas schlechtere aber dennoch ausreichend gute Abbildungsqualität, ist dafür aber viel schneller. Die Artefakte sind jedoch nicht so klar abgebildet, so daß sie sich für schnelle Übersichtsaufnahmen exzellent eignet. Die EPI-Sequenz ist nur etwas schneller als die FFE-, hat aber eine viel geringere Abbildungsqualität und höhere Artefaktanfälligkeit.

Mit den Messungen und Berechnungen konnte nachgewiesen werden, daß Werkstoffflanken die senkrecht zum Magnetfeld stehen vergleichsweise größere Feldinhomogenitäten hervorrufen als parallele, und daß die Enden von Proben starke, entgegengesetzte Spitzen der Feldveränderung erzeugen, die sich aber relativ schnell mit der Entfernung abbauen. Genau dieses Phänomen macht eine vollständige Kompensation der Feldinhomogenitäten mit dem untersuchten Ansatz schwer. Es konnte belegt werden, daß der analytische Ansatz richtig ist. Er gilt aber nur theoretisch, für unendlich lange, senkrecht zum Feld stehende Doppelzylinder. Da die Doppelzylinder in der Realität endlich sind und somit ebenfalls die andere Schnittebene (entlang der Achse) Einfluß auf das Feld nimmt, ist diese gleichfalls zu berücksichtigen. Mit dem verwendeten zweidimensionalen finite Elemente Programm ist die gegenseitige Beeinflussung dieser Betrachtungsebenen nur sehr schwer abzuschätzen. Es konnte aber gezeigt werden, daß bei endlichen Doppelzylindern eine Optimierung der einen Schnittebene eine Verschlechterung der anderen zur Folge hat. Bei den in dieser Arbeit verwendeten AbmaBen der Versuchsproben (Vorversuche: $50 \mathrm{~mm}$ lang und 20mm Durchmesser; Kompensationsmessungen: ähnliche Dimensionen, je nach Berechnung) wurde ersichtlich, daß transversal der Einfluß der Probenenden größer als der vom Probenquerschnitt herrührende ist. Sagittal sind nur die von den Probenenden verursachten Feldinhomogenitäten ausschlaggebend. Zur Berechnung ciner Kompensation eignet sich sagittal also eine zweidimensionale Betrachtungsweise. Um aber genaue Ergebnisse zu erhalten, speziell für die transversale Ausrichtung, ist eine dreidimensionale Betrachtungsweise notwendig Es bleibt fragwürdig, ob man eine Kombination findet. die auch die Extremwerte der direkt an den Probenenden entstehenden Feldverzerrungen so weit minımieren kann, daß sie keine Artefakte bei den Messungen im MRT mehr hervorrufen.

Die Betrachtungen der Messungen haben gezcigt, dall ab $10^{-6}$ Tesla Feldabweichung signitikante Artefahtc auftreten.

Aus den oben genannten Gründen ist es nicht gelungen für dic transversale Orientienung der Doppeliylinder 
cine vollständige Kompensation zu crreichen. Dic Artefakte, dic separate Werkstoffe transversal hervorrufen konnten aber tcilweise durch cinc Kombination schr reduziert und sngittal vollständig kompensiert werden.
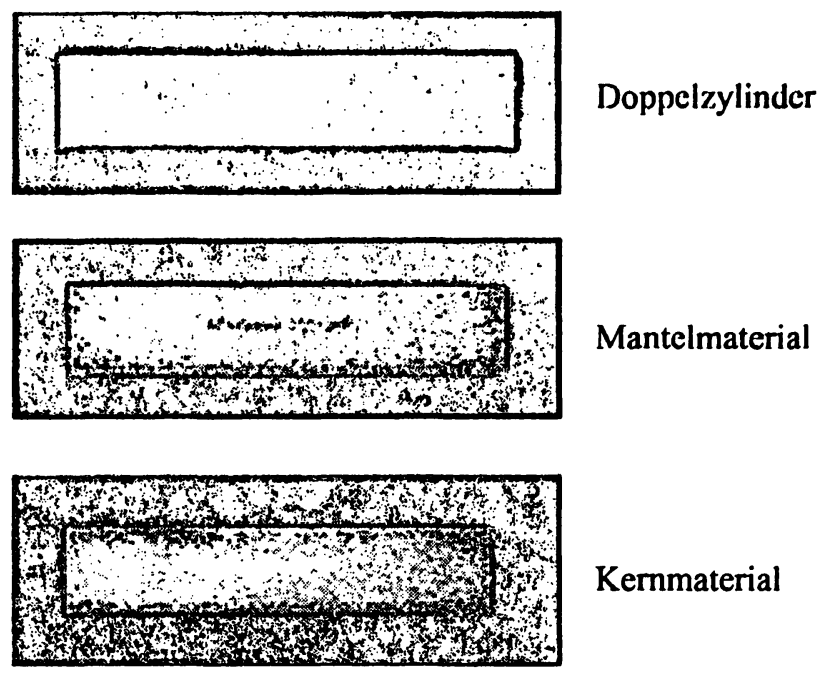

Abbildung 5: Kompensationsmessung

Die Abbildung 5 zeigt als Beispiel einer Kompensation Messungen mit der FFE-Sequenz. Es wurde ein Doppelzylinder verwendet, bestehend aus einem Aluminiummantel (Länge: 51,3mm; Außendurchmesser: 5,4mm; Innendurchmesser: $3 \mathrm{~mm}$ ) und einem $\mathrm{Al}_{2} \mathrm{O}_{3}$ Kern (Länge 51,3mm; Durchmesser: $3 \mathrm{~mm}$ ). Es wurden drei Vergleichsmessungen durchgeführt und mit der Referenzmessung verglichen. Bei der Messung mit dem Doppelzylinder sind kein Artefakte mehr sichtbar. Die Messungen mit den separaten Materialien weisen jedoch noch welche auf, die ein entgegengesetztes Artefaktverhalten zeigen. Bei der Abbildung des paramagnetischen Aluminiummantels sind Verdunkelungen in Form der Probe zu erkennen und bei dem diamagnetischen Kernmaterial Aufhellungen.

\section{Diskussion}

Der in dieser Arbeit untersuchte Ansatz zur Kompensation von Feldinhomogenitäten mit Doppelzylindern aus para- und diamagnetischen Werkstoffen hat gezeigt, daß eine Realisierung mit sehr vielen Problemen behaftet ist. Die bisherigen Ergebnisse können das erwartete Ziel nicht erfüllen, auch wenn Erfolge in der Kompensation zu verzeichnen waren.

Bei einer weiteren Verfolgung dieses Ansatzes empfiehlt sich die Verwendung eines Rechenprogrammes, das eine dreidimensionale Betrachtungsweise gewährleistet. Des weiteren sollte als Mantelmaterial ein paramagnetischer Werkstoff gewählt werden, da so der eventuelle Einfluß von Wirbelstromartefakten minimiert wird, und die verbleibenden Artefakte vom Mantelmaterial dominiert werden, also eine Aufhellung des Bildes zur Folge haben. Bildaufhellungen haben eine geringere Wirkung auf die Abbildungsqualität.
Jc länger der Doppelzylinder ist desto kleiner wird der Einfluß der von den Enden hervorgerufenen Feldveränderungen bezogen auf die gesamte Probe. Dann kann auch die vom Querschnitt verursachte Feldstörung weiter minimiert werden. Für Materialien, die das Magnetfeld des MRT vollständig und senkrecht durchqueren, kann cine Bercchnung der Durchmesserverhältnisse nach dem analytischen Ansatz angewendet werden. Ist das zu messende Objekt klciner als ein sagittal ausgerichtetes Werkstück, ist eine Minimierung der Feldinhomogenitäten im zwcidimensionalen Längsschnitt ausreichend.

Eine bedachte Kombination von dia- und paramagnetischen Werkstoffen ist allgemein sinnvoll, da eine Kompensation der Feldinhomogenitäten eintritt. Der analytische Ansatz eignet sich dafür als Richtlinie und hat zumindest eine Verkürzung des Mindestabstandes (Entfernung bis keine Artefakte mehr auftreten) oder der Eindringtiefe zur Folge.

Allgemein weist dieser Ansatz allerdings sehr große Defizite auf. Auch wenn eine ausreichend gute Kompensation der von allen Dimensionen verursachten Feldinhomogenitäten gefunden wird, müßte für alle unterschiedlichen Abmaße der Zylinder eine neue umfangreiche Berechnung erfolgen, solange keine allgemeingültige Formel zur Berechnung der Durchmesserverhältnisse für alle Längen und Gesamtdurchmesser bei bekannten Suszeptibilitäten zur Verfügung steht. Die Messungen der Suszeptibilitäten haben gezeigt, daß namentlich gleiche Materialien unterschiedliche Suszeptibilitäten aufweisen können. Die Werte variieren also je nach genauer Zusammensetzung in einer großen Bandbreite. Es wäre somit nötig nur reine Werkstoffe zu verwenden oder Materialien ausfindig zu machen, deren Suszeptibilitäten konstant sind.

Erfüllt man diese Bedingungen sind die gefundenen Werkstoffkombinationen an die zylindrische Geometrie und die transversale oder sagittale Orientierung im MRT gebunden.

Ein interessanterer und sinnvollerer Ansatz wäre also ein Material zu finden oder aus dia- und paramagnetischen Grundwerkstoffen zu kreieren, das von sich aus keine Suszeptibilitätsartefakte verursacht und eine ausreichende Festigkeit aufweist. Ein derartiger Werkstoff könnte universell im MRT eingesetzt werden und würde nicht geometrischen Vorgaben oder bestimmten Orientierungen im Magnetfeld obliegen.

\section{Literaturverzeichnis}

[1] J. D. Jackson, Klassische Elektrodynamik 2. Auflage, Berlin New York: de Gruyter, 1982; zitiert bei: S. Fritzsche, R. Thull, A. Haase, Biomedizinische Technik vol. 38; Ergänzungsband, p. 115, 1993 